\title{
Hybrid Group Acceptance Sampling Plan Based on Size Biased Lomax Model
}

\author{
R. Subba Rao ${ }^{1, *}$, A. Naga Durgamamba ${ }^{2}$, R.R.L. Kantam ${ }^{3}$ \\ ${ }^{1}$ Shri Vishnu Engineering College for Women, Bhimavaram, 534202, Andhra Pradesh, India \\ ${ }^{2}$ Raghu Institute of Technology, Dakamarri, Visakhapatnam, 531162, Andhra Pradesh, India \\ ${ }^{3}$ Acharya Nagarjuna University, Guntur, 522510, Andhra Pradesh, India \\ *Corresponding Author: rsr_vishnu@rediffmail.com
}

Copyright (C) 2014 Horizon Research Publishing All rights reserved.

\begin{abstract}
In this paper, a hybrid group acceptance sampling plan is introduced for a truncated life test if life times of the items follow size biased Lomax model. The minimum number of testers and acceptance number are obtained when the consumer's risk and the test termination time and group size are pre-specified. The operating characteristic values, minimum ratios of the true mean life to the specified mean life for the given producer's risk are also derived. The results are discussed through an example, a comparative study of proposed sampling plan with existing sampling plan are elaborated.
\end{abstract}

Keywords Size Biased Lomax Model (SBLM), Group Acceptance Sampling Plan (GASP), Consumer's Risk, Producer's Risk, Operating Characteristic (O.C), Truncated Life Test

\section{Introduction}

A decision on accepting or rejecting a product depends on its fitness for use. The quality checking process in quality control is of different types. One such process is acceptance sampling plans. In most acceptance sampling plans, the major problem is to determine the sample size from a lot under consideration. In usual sampling plan the decision of accepting or rejecting a lot on the basis of a single item. The acceptance sampling plan that accommodates multiple number of items at a time put in a tester will be called a group acceptance sampling plan, which reduces the testing time as well as cost can be saved by testing those items simultaneously. While designing a group acceptance sampling plan determining the sample size is equivalent to determine the number of groups as the number of testers is known. The experiment is truncated if more than the number of failures occurred any group during the experiment time. The recent technique that is incorporate in this paper is hybrid group acceptance sampling plan, in which the minimum number of testers will be obtain for a pre-determined number of groups. Hybrid group acceptance sampling plan is based on truncated life test assuming that the life time of a product follows a specific probability model.

The attention made by different researchers on the development of Hybrid Group Acceptance Sampling Plan (HGASP) and its models are: G. Srinivas Rao[7,8] developed a hybrid group acceptance sampling plans for lifetimes based on log-logistic distribution and a hybrid group acceptance sampling plan for lifetimes based on generalized exponential distribution, A. Baklize[1] studied acceptance sampling based on truncated life tests in the Pareto distribution of the second kind, Muhammad Aslam et al.[3] presented a group acceptance plan based on truncated life test for Gamma distribution, K. Rosaiah et al. [6,7] given an economic Reliability test plan with Pareto distribution and Pareto distribution in acceptance sampling based on truncated life test, A.R. Sudamani Ramaswamy[10] determined a hybrid group acceptance sampling plans for lifetimes based on exponentiated weibull distribution, Jaffer Hussain et al.[2] derived a hybrid group acceptance sampling plans for lifetimes having generalized Pareto distribution, K. Rosaiah and R.R.L. Kantam[4] who derived acceptance sampling based on the inverse Rayleigh distribution, K. Rosaiah et al.[5] discussed Reliability of test plans for exponentiated log-logistic distribution.

In this paper a new hybrid group acceptance sampling plan (HGASP) is proposed by considering a size biased Lomax model with known shape parameter. The probability density function(p.d.f) $f(\mathrm{t})$ and cumulative distribution function(c.d.f) $F(t)$ of size biased Lomax model are given below

$$
\begin{aligned}
f(\mathrm{t})=\frac{\alpha(\alpha-1)}{\sigma} \frac{t}{\sigma}\left(1+\frac{t}{\sigma}\right)^{-(\alpha+1)} ; t \geq 0, \alpha \succ 1, \sigma \succ 0 \\
\mathrm{~F}(\mathrm{t})=1-\left(1+\frac{\alpha t}{\sigma}\right)\left(1+\frac{t}{\sigma}\right)^{-\alpha} ; t \geq 0, \alpha \succ 1 \\
\sigma \succ 0
\end{aligned}
$$

Where $\alpha$ is shape and $\sigma$ is scale parameter. Mean and variance of size biased Lomax model are given by 


$$
E(t)=\frac{2 \sigma}{\alpha-2} ; \alpha \succ 2 \text { and } V(t)=\frac{2 \alpha \sigma^{2}}{(\alpha-2)^{2}(\alpha-3)} ; \alpha \succ 3
$$

Construction of hybrid group acceptance sampling plan for size biased Lomax model is presented in Section 2. The operating characteristic values are given in Section 3. Description of tables and examples are discussed in Section 4. Concluding remarks are given in Section 5.

\section{Design of the Proposed Sampling Plan}

Let $\mu$ be the true mean life of a product and $\mu_{0}$ is the specified mean life of an item, under the assumption that the lifetime of an item follows size biased Lomax model. If $\mathrm{H}_{0}: \mu \geq \mu_{0}$, the lot of the product is accepted, otherwise the lot of the product is rejected. In acceptance sampling schemes, this hypothesis is tested on the basis of number of failures in a sample with pre-fixed time.

HGASP follows the following steps:

$\checkmark \quad$ Select the number of testers, $r$ and assign the $r$ items to each predefined groups, $\mathrm{g}$, the required sample size for a lot is $n=r g$.

$\checkmark \quad$ Pre-fix the acceptance number, $\mathrm{c}$ for each group and the experiment time $t_{0}$.

$\checkmark$ Accept the lot if at most $\mathrm{c}$ failures occurs in each of all groups.

$\checkmark$ Terminate the experiment if more than c failures occur in any group and reject the lot.

Determine the number of tester's $r$, for size biased Lomax model and various values of acceptance number $\mathrm{c}$, whereas the number of groups $g$, and the termination time $t_{0}$ are assumed to be specified. It is convenient to consider that termination time as a multiple of the specified mean life $\mu_{0}$, consider $\mathrm{t}_{0}=a$. $\mu_{0}$, where ' $\mathrm{a}$ ' is a constant termination ratio.

The probability of rejecting a good lot is called the producer's risk and the probability of accepting a bad lot is called the consumer's risk and are respectively ' $\gamma$ ' and ' $\beta$ '. The parameter value of $r$ of the proposed sampling plan is derived by consumer's risk $\beta$. If the confidence level is $\mathrm{p}^{*}$, then $\beta=1-p^{*}$. If the lot size is large enough, we can use binomial distribution to develop the HGASP. The lot of the product is accepted only if every group $g$ having at most $c$ failures. The HGASP is characterized by the three parameters $\left(\mathrm{n}, \mathrm{c}, \mathrm{t} / \sigma_{0}\right)$. The lot acceptance probability is

$$
L(p)=\left[\sum_{i=o}^{c} r_{c_{i}} p^{i}(1-p)^{r-i}\right]^{g}
$$

where $\mathrm{p}$ is the probability that an item in a tester fails before the termination time $t_{0}=a . \mu_{0}$ and is given by

$$
\mathrm{p}=\mathrm{F}(\mathrm{t})=1-\left(1+\frac{2 \alpha a}{\left(\frac{\mu}{\mu_{0}}\right)(\alpha-2)}\right)\left(1+\frac{2 a}{\left(\frac{\mu}{\mu_{0}}\right)(\alpha-2)}\right)^{-\alpha}
$$

The minimum number of testers $\mathrm{r}$ can be derived by considering the consumer's risk when the true mean life equals to specified mean life $\left(\mu=\mu_{0}\right)$ through the following inequality

$$
L\left(p_{0}\right) \leq \beta
$$

where $\mathrm{p}_{0}$ is the failure probability at $\mu=\mu_{0}$, and it is given by

$$
\mathrm{p}=\mathrm{F}(\mathrm{t})=1-\left(1+\frac{2 \alpha a}{(\alpha-2)}\right)\left(1+\frac{2 a}{(\alpha-2)}\right)^{-\alpha}
$$

Table 1 show for the pre-fix consumer's risk $(\beta)$, number of groups (g), acceptance number (c) and termination time (a) to obtain the minimum testers ( $\mathrm{r}$ ). The minimum number of testers required for the size biased Lomax model at $\alpha=3$

\begin{tabular}{|c|c|c|c|c|c|c|c|c|}
\hline \multirow{2}{*}{$\beta$} & \multirow{2}{*}{$\mathrm{g}$} & \multirow{2}{*}{ c } & \multicolumn{6}{|c|}{$a$} \\
\hline & & & 0.7 & 0.8 & 1.0 & 1.2 & 1.5 & 2.0 \\
\hline \multirow{8}{*}{0.25} & 2 & 0 & 1 & 1 & 1 & 1 & 1 & 1 \\
\hline & 3 & 1 & 2 & 2 & 2 & 2 & 2 & 2 \\
\hline & 4 & 2 & 4 & 3 & 3 & 3 & 3 & 3 \\
\hline & 5 & 3 & 5 & 5 & 4 & 4 & 4 & 4 \\
\hline & 6 & 4 & 6 & 6 & 5 & 5 & 5 & 5 \\
\hline & 7 & 5 & 7 & 7 & 7 & 6 & 6 & 6 \\
\hline & 8 & 6 & 9 & 8 & 8 & 7 & 7 & 7 \\
\hline & 9 & 7 & 10 & 10 & 9 & 9 & 8 & 8 \\
\hline \multirow{8}{*}{0.10} & 2 & 0 & 2 & 2 & 1 & 1 & 1 & 1 \\
\hline & 3 & 1 & 3 & 3 & 2 & 2 & 2 & 2 \\
\hline & 4 & 2 & 4 & 4 & 4 & 3 & 3 & 3 \\
\hline & 5 & 3 & 5 & 5 & 5 & 4 & 4 & 4 \\
\hline & 6 & 4 & 7 & 6 & 6 & 6 & 5 & 5 \\
\hline & 7 & 5 & 8 & 8 & 7 & 7 & 6 & 6 \\
\hline & 8 & 6 & 10 & 9 & 8 & 8 & 7 & 7 \\
\hline & 9 & 7 & 11 & 10 & 10 & 9 & 9 & 8 \\
\hline \multirow{3}{*}{0.05} & 2 & 0 & 2 & 2 & 2 & 1 & 1 & 1 \\
\hline & 3 & 1 & 3 & 3 & 3 & 3 & 2 & 2 \\
\hline & 4 & 2 & 5 & 4 & 4 & 4 & 3 & 3 \\
\hline
\end{tabular}
are calculated and are given in Table 2.

Table 1. The pre-fix values of $\beta, g, c$ and $a$

\begin{tabular}{|c|c|c|c|}
\hline$\beta$ & $\mathrm{g}$ & $\mathrm{c}$ & $a$ \\
\hline 0.25 & 2 & 0 & 0.7 \\
\hline 0.10 & 3 & 1 & 0.8 \\
\hline 0.05 & 4 & 2 & 1.0 \\
\hline 0.01 & 5 & 3 & 1.2 \\
\hline \multirow{5}{*}{} & 6 & 4 & 1.5 \\
\cline { 2 - 4 } & 7 & 5 & 2.0 \\
\cline { 2 - 4 } & 8 & 6 & \\
\cline { 2 - 4 } & 9 & 7 & \\
\hline
\end{tabular}

Table 2. Number of testers required for the proposed plan SBLM at $\alpha=3$ 


\begin{tabular}{|c|c|c|c|c|c|c|c|c|}
\hline \multirow{5}{*}{} & 5 & 3 & 6 & 5 & 5 & 5 & 4 & 4 \\
\cline { 2 - 9 } & 6 & 4 & 7 & 7 & 6 & 6 & 5 & 5 \\
\cline { 2 - 9 } & 7 & 5 & 8 & 8 & 7 & 7 & 6 & 6 \\
\hline & 8 & 6 & 10 & 9 & 9 & 8 & 8 & 7 \\
\hline & 9 & 7 & 11 & 11 & 10 & 9 & 9 & 8 \\
\hline \multirow{5}{*}{0.01} & 2 & 0 & 3 & 3 & 2 & 2 & 2 & 2 \\
\cline { 2 - 9 } & 3 & 1 & 4 & 4 & 3 & 3 & 3 & 2 \\
\hline & 4 & 2 & 5 & 5 & 4 & 4 & 4 & 3 \\
\hline & 5 & 3 & 7 & 6 & 5 & 5 & 5 & 4 \\
\hline & 7 & 5 & 9 & 9 & 8 & 7 & 7 & 6 \\
\hline & 8 & 6 & 11 & 10 & 9 & 9 & 8 & 8 \\
\hline & 9 & 7 & 12 & 11 & 10 & 10 & 9 & 9 \\
\hline
\end{tabular}

\section{Operating Characteristic}

The Operating characteristic function of a sampling plan is the probability of acceptance i.e., a function of the deviation of specified mean $\mu_{0}$ from its true mean $\mu$. Once the minimum sample size is obtained, one may be interested to find the probability of acceptance of a lot when the quality of the product is good enough. The product is considered to be good if $\mu \geq \mu_{0}$. The probabilities based on (4) for various mean life times $\left(\mu / \mu_{0}=2,4,6,8,10,12\right)$ under $\beta=0.25,0.10,0.05,0.01$ with the termination ratio $a$ $=0.7,0.8,1.0,1.2,1.5,2.0$ choosing acceptance number $\mathrm{c}=$ 2 are presented in Table 3.

For given producer's risk the minimal ratios of true mean life to the specified mean life can be obtained by using the inequality

$$
\left[\sum_{i=0}^{c} r_{c_{i}} p^{i}(1-p)^{r-i}\right]^{g} \geq 1-\alpha
$$

where $\mathrm{p}$ is given in (5) and for various combinations of consumer's risk $\beta, a$ and $\gamma=0.05$, the minimum mean ratio's are found for fixed values of $\mathrm{c}$ and are given in Table 4.

Table 3. O.C values of the HGASP with $\mathrm{g}=4$ and $\mathrm{c}=2$ for SBLM with $\alpha=3$.

\begin{tabular}{|c|c|c|c|c|c|c|c|c|}
\hline \multirow{2}{*}{$\beta$} & \multirow{2}{*}{$\mathrm{r}$} & \multirow{2}{*}{$a$} & \multicolumn{6}{|c|}{$\mu / \mu_{0}$} \\
\hline & & & 2 & 4 & 6 & 8 & 10 & 12 \\
\hline \multirow{6}{*}{0.25} & 4 & 0.7 & 0.5335 & 0.9366 & 0.9878 & 0.9967 & 0.9989 & 0.9996 \\
\hline & 3 & 0.8 & 0.7400 & 0.9692 & 0.9940 & 0.9984 & 0.9994 & 0.9998 \\
\hline & 3 & 1.0 & 0.5862 & 0.9321 & 0.9848 & 0.9955 & 0.9984 & 0.9993 \\
\hline & 3 & 1.2 & 0.4450 & 0.8792 & 0.9692 & 0.9902 & 0.9963 & 0.9984 \\
\hline & 3 & 1.5 & 0.2807 & 0.7775 & 0.9321 & 0.9759 & 0.9902 & 0.9955 \\
\hline & 3 & 2.0 & 0.1241 & 0.5862 & 0.8366 & 0.9321 & 0.9692 & 0.9848 \\
\hline \multirow{6}{*}{0.10} & 4 & 0.7 & 0.5335 & 0.9366 & 0.9878 & 0.9967 & 0.9989 & 0.9996 \\
\hline & 4 & 0.8 & 0.4109 & 0.8980 & 0.9783 & 0.9939 & 0.9979 & 0.9991 \\
\hline & 4 & 1.0 & 0.2234 & 0.7936 & 0.9472 & 0.9835 & 0.9939 & 0.9974 \\
\hline & 3 & 1.2 & 0.4450 & 0.8792 & 0.9692 & 0.9902 & 0.9963 & 0.9984 \\
\hline & 3 & 1.5 & 0.2807 & 0.7775 & 0.9321 & 0.9759 & 0.9902 & 0.9955 \\
\hline & 3 & 2.0 & 0.1241 & 0.5862 & 0.8366 & 0.9321 & 0.9692 & 0.9848 \\
\hline \multirow{6}{*}{0.05} & 5 & 0.7 & 0.2912 & 0.8651 & 0.9717 & 0.9922 & 0.9973 & 0.9989 \\
\hline & 4 & 0.8 & 0.4109 & 0.8980 & 0.9783 & 0.9939 & 0.9979 & 0.9991 \\
\hline & 4 & 1.0 & 0.2234 & 0.7936 & 0.9472 & 0.9835 & 0.9939 & 0.9974 \\
\hline & 4 & 1.2 & 0.1126 & 0.6660 & 0.8980 & 0.9650 & 0.9862 & 0.9939 \\
\hline & 3 & 1.5 & 0.2807 & 0.7775 & 0.9321 & 0.9759 & 0.9902 & 0.9955 \\
\hline & 3 & 2.0 & 0.1241 & 0.5862 & 0.8366 & 0.9321 & 0.9692 & 0.9848 \\
\hline \multirow{6}{*}{0.01} & 5 & 0.7 & 0.2912 & 0.8651 & 0.9717 & 0.9922 & 0.9973 & 0.9989 \\
\hline & 5 & 0.8 & 0.1816 & 0.7922 & 0.9510 & 0.9856 & 0.9949 & 0.9979 \\
\hline & 4 & 1.0 & 0.2234 & 0.7936 & 0.9472 & 0.9835 & 0.9939 & 0.9974 \\
\hline & 4 & 1.2 & 0.1126 & 0.6660 & 0.8980 & 0.9650 & 0.9862 & 0.9939 \\
\hline & 4 & 1.5 & 0.0377 & 0.4703 & 0.7936 & 0.9186 & 0.9650 & 0.9835 \\
\hline & 3 & 2.0 & 0.1241 & 0.5862 & 0.8366 & 0.9321 & 0.9692 & 0.9848 \\
\hline
\end{tabular}


Table 4. Minimum ratio values of true mean life to specified mean life for $\gamma=0.05$ for SBLM at $\alpha=3$.

\begin{tabular}{|c|c|c|c|c|c|c|c|c|}
\hline \multirow{2}{*}{$\beta$} & \multirow{2}{*}{$\mathrm{g}$} & \multirow{2}{*}{$\mathrm{c}$} & \multicolumn{6}{|c|}{$a$} \\
\hline & & & 0.7 & 0.8 & 1.0 & 1.2 & 1.5 & 2.0 \\
\hline \multirow{8}{*}{0.25} & 2 & 0 & 13.35 & 15.26 & 19.07 & 22.89 & 28.61 & 38.14 \\
\hline & 3 & 1 & 4.8 & 5.48 & 6.85 & 8.22 & 10.28 & 13.7 \\
\hline & 4 & 2 & 4.27 & 3.5 & 4.38 & 5.25 & 6.57 & 8.75 \\
\hline & 5 & 3 & 3.18 & 6.64 & 3.32 & 3.99 & 4.98 & 6.64 \\
\hline & 6 & 4 & 2.58 & 2.95 & 2.73 & 3.28 & 4.1 & 5.46 \\
\hline & 7 & 5 & 2.2 & 2.51 & 3.14 & 2.82 & 3.52 & 4.7 \\
\hline & 8 & 6 & 2.27 & 2.16 & 2.69 & 2.44 & 3.04 & 4.06 \\
\hline & 9 & 7 & 1.99 & 2.28 & 2.37 & 2.85 & 2.7 & 3.59 \\
\hline \multirow{8}{*}{0.10} & 2 & 0 & 19.6 & 22.4 & 19.07 & 22.89 & 28.61 & 38.14 \\
\hline & 3 & 1 & 6.82 & 7.8 & 6.85 & 8.22 & 10.28 & 13.7 \\
\hline & 4 & 2 & 4.27 & 4.87 & 6.09 & 5.25 & 6.57 & 8.75 \\
\hline & 5 & 3 & 3.18 & 3.64 & 4.55 & 3.99 & 4.98 & 6.64 \\
\hline & 6 & 4 & 3.13 & 2.95 & 3.69 & 4.42 & 4.1 & 5.46 \\
\hline & 7 & 5 & 2.65 & 3.03 & 3.14 & 3.77 & 3.52 & 4.7 \\
\hline & 8 & 6 & 2.6 & 2.59 & 2.69 & 3.23 & 3.04 & 4.06 \\
\hline & 9 & 7 & 2.28 & 2.28 & 2.84 & 2.85 & 3.56 & 3.59 \\
\hline \multirow{8}{*}{0.05} & 2 & 0 & 19.6 & 22.4 & 28 & 22.89 & 28.61 & 38.14 \\
\hline & 3 & 1 & 6.82 & 7.8 & 9.74 & 11.69 & 10.28 & 13.7 \\
\hline & 4 & 2 & 5.23 & 4.87 & 6.09 & 7.31 & 6.57 & 8.75 \\
\hline & 5 & 3 & 3.88 & 3.64 & 4.55 & 5.45 & 4.98 & 6.64 \\
\hline & 6 & 4 & 3.13 & 3.57 & 3.69 & 4.42 & 4.1 & 5.46 \\
\hline & 7 & 5 & 2.65 & 3.03 & 3.14 & 3.77 & 3.52 & 4.7 \\
\hline & 8 & 6 & 2.6 & 2.59 & 3.24 & 3.23 & 4.04 & 4.06 \\
\hline & 9 & 7 & 2.28 & 2.61 & 2.84 & 2.85 & 3.56 & 3.59 \\
\hline \multirow{8}{*}{0.01} & 2 & 0 & 24.41 & 27.89 & 28 & 33.6 & 42 & 56 \\
\hline & 3 & 1 & 8.43 & 9.63 & 9.74 & 11.69 & 14.61 & 13.7 \\
\hline & 4 & 2 & 5.23 & 5.98 & 6.09 & 7.31 & 9.13 & 8.75 \\
\hline & 5 & 3 & 4.48 & 4.43 & 4.55 & 5.45 & 6.82 & 6.64 \\
\hline & 6 & 4 & 3.61 & 3.57 & 4.47 & 4.42 & 5.53 & 5.46 \\
\hline & 7 & 5 & 3.05 & 3.48 & 3.78 & 3.77 & 4.71 & 4.7 \\
\hline & 8 & 6 & 2.91 & 2.97 & 3.24 & 3.88 & 3.56 & 4.74 \\
\hline & 9 & 7 & 2.55 & 2.61 & 2.84 & 3.41 & 3.56 & 4.74 \\
\hline
\end{tabular}

\section{Description of Tables and Examples}

The require parameters of HGASP are evaluated at various values of the consumer's risk and the termination time in Table 2 . The minimum sample size is calculated by using the relation $n=r g$. Table 2 indicates that, as the test termination time $a$ increases, the number of testers $\mathrm{r}$ either decrease or constant, i.e., a smaller number of testers is needed, if the termination time increases at a fixed number of groups. Suppose, from Table w, if $\beta=0.25, \mathrm{~g}=4, \mathrm{c}=2$ and a changes from 0.7 to 0.8 , the required values of design parameters of HGASP changes from $r=4$ to $r=3$. The probability of acceptance for the lot at the mean ratio corresponding to the producer's risk is presented in Table 3.

For an example, the lifetime of a product follows the size biased Lomax distribution with $a=3$. It is desired to design a HGASP to test if the mean life is greater than $1000 \mathrm{hrs}$ based on a testing time of $700 \mathrm{hrs}$ and using 4 groups. Thus, we will draw a random sample of size $16(\mathrm{n}=\mathrm{r}$. g) items and allocate 4 items to each of 4 groups to put on test for $700 \mathrm{hrs}$. We will accept the lot if no more than 2 failures occurs before $700 \mathrm{hrs}$ in each of 4 groups. We truncate the experiment as soon as the $3^{\text {rd }}$ failure occurs before the $700^{\text {th }}$ $\mathrm{hr}$. 
Table 5. At $\mathrm{r}=4$ and $\mathrm{a}=0.7$, O.C values of the HGASP with $\mathrm{g}=4$ and $\mathrm{c}=2$ for SBLM with $\alpha=3$.

\begin{tabular}{|c|c|c|c|c|c|c|}
\hline$\mu / \mu_{0}$ & 2 & 4 & 6 & 8 & 10 & 12 \\
\hline $\mathrm{p}_{a}$ & .5335 & .9366 & .9878 & .9967 & .9989 & .9996 \\
\hline
\end{tabular}

Table 5 explains (borrowed from table 3 ) that, if the true mean life is 4 times of $1000 \mathrm{hrs}$, the producer's risk is 0.0634 . So, a lot of submitted items shall be accepted with probability 0.5335 if the true mean life is 2 times the specified mean life. The acceptance probability of submitted lot is increased up to 0.9996 if the true mean life of an item in a lot is 12 times the specified mean life.

In order to compare proposed HGASP with that of G. Srinivas Rao(2012), we consider the above example. The HGASP for the log-logistic distribution are $(\mathrm{g}, \mathrm{r}, \mathrm{c}, a)=$ $(4,10,2,0.7)$ with $\delta=3$ and the HGASP for proposed sampling plan are $(\mathrm{g}, \mathrm{r}, \mathrm{c}, a)=(4,4,2,0.7)$. So, our proposed HGASP requires $16(\mathrm{n}=\mathrm{r}$. g) items where as the existing plan given by G. Srinivas Rao(2012) requires 32 items respectively to reach on a same decision about submitted items. A comparison of sample sizes for both the plans with $\beta=0.10$ are exhibited in Table 6 .

Table 6. Comparisons of sample size (n) when $\mathrm{g}=4$ and $\mathrm{c}=2$

\begin{tabular}{|c|c|c|c|}
\hline $\mathrm{a}$ & $\beta$ & $\begin{array}{l}\text { Existing } \\
\text { HGASP }\end{array}$ & $\begin{array}{c}\text { Proposed } \\
\text { HGASP } \\
\end{array}$ \\
\hline 0.7 & \multirow{6}{*}{0.10} & 10 & 4 \\
\hline 0.8 & & 7 & 4 \\
\hline 1.0 & & 5 & 4 \\
\hline 1.2 & & 4 & 3 \\
\hline 1.5 & & 3 & 3 \\
\hline 2.0 & & 3 & 3 \\
\hline
\end{tabular}

\section{Conclusion}

In this paper, a hybrid group acceptance sampling plan from the truncated life test was proposed, the number of testers and the acceptance number was derived for size biased Lomax model with $\alpha=3$ when the consumer's risk $(\beta)$ and other parameters specified. It can be observed that the minimum number of testers required is decreases as test termination time increases and also the operating characteristics values increases more rapidly as the quality improves. Hybrid group acceptance sampling plan is more preferable than group acceptance sampling plan. The evaluated values of ' $\mathrm{r}$ ' for a given ' $\mathrm{g}$ ' by hybrid group acceptance sampling plan is showing a stability resulting ' $n$ ' usage that a common table at some stage for all values of ' $\alpha$ ' whereas in group acceptance sampling plan the derived values of ' $\mathrm{g}$ ' for a given ' $\mathrm{r}$ ' are increasing rapidly for various values of ' $\alpha$ '. If the data follows size biased Lomax mode, our proposed sampling plan of HGASP gives more efficient results than existing sampling plan.

\section{REFERENCES}

[1] A. Baklize. Acceptance sampling based on truncated life tests in the Pareto distribution of the second kind. Advances and Applications in Statistics, 3(1), 33-48. (2003).

[2] Jaffer Hussain, Abdur Razzaque Mughal, Muhammad Khalid Pervaiz and Usman Ali. A hybrid group acceptance sampling plans for lifetimes having generalized Pareto distribution, Journal of Statistics, vol 19, 31-42, (2012).

[3] Muhammad Aslam, Chi-Hyuck Jun and Munir Ahmad. A group acceptance plan based on truncated life test for Gamma distribution, Pak. J. Statist., vol. 25(3), 333-340, (2009).

[4] K. Rosaiah and R.R.L. Kantam. Acceptance sampling based on the inverse Rayleigh distribution, Economic Quality Control 20(2), 277-286, (2005).

[5] K. Rosaiah, R.R.L. Kantam and Ch. Santosh Kumar. Reliability of test plans for exponentiated log-logistic distribution, Economic Quality Control, 21(2), 165-175, (2006).

[6] K. Rosaiah, R.R.L. Kantam and R. Subba Rao. An economic Reliability test plan with Pareto distribution, International Journal of Agricultural Statistical Science, 3(2), 309-317, (2007).

[7] K. Rosaiah, R.R.L. Kantam and R. Subba Rao. Pareto distribution in acceptance sampling based on truncated life tests. IAPQR Transactions Vol. 34, No. 1, (2009).

[8] G. Srinivas Rao. A hybrid group acceptance sampling plans for lifetimes based on log-logistic distribution, Journal of Reliability and Statistical Studies, 4(1), 31-40, (2011).

[9] G. Srinivas Rao. A hybrid group acceptance sampling plan for lifetimes based on generalized exponential distribution, Journal of Applied Sciences, 11(12), 2232-2237, (2011).

[10] A.R. Sudamani Ramaswamy. A hybrid group acceptance sampling plans for lifetimes based on exponentiated weibull distribution, International Journal of Mathematical Archives, 3(10), 3777-3782, (2012). 